\title{
Circuit
}

Musiques contemporaines

\section{Introduction : la voix d'Ana Sokolović}

\section{Jonathan Goldman}

Volume 22, numéro 3, 2012

Viva la musica ! Ana Sokolović

URI : https://id.erudit.org/iderudit/1014224ar

DOI : https://doi.org/10.7202/1014224ar

Aller au sommaire du numéro

Éditeur(s)

Les Presses de l’Université de Montréal

ISSN

1183-1693 (imprimé)

1488-9692 (numérique)

Découvrir la revue

Citer ce document

Goldman, J. (2012). Introduction : la voix d'Ana Sokolović. Circuit, 22(3), 5-7. https://doi.org/10.7202/1014224ar d'utilisation que vous pouvez consulter en ligne.

https://apropos.erudit.org/fr/usagers/politique-dutilisation/ 


\section{Introduction : la voix d'Ana Sokolović}

Jonathan Goldman

Tous les deux ans depuis 2008, sur l'initiative de son directeur artistique Walter Boudreau, la Société de musique contemporaine du Québec (SMCQ) consacre sa saison artistique à la démarche d'un seul créateur. Dans le cadre de cette Série hommage, la SMCQ invite des dizaines d'organismes de musique contemporaine à travers le Canada à s'unir à elle dans une joyeuse profusion d'événements qui comprend un axe pédagogique important en collaboration avec le système scolaire. La clé du succès de cet effort repose sur l'impressionnante force fédératrice de la SMCQ et sur la volonté d'autres organismes de s'associer avec elle pour créer un événement majeur qui s'étend sur plus d'un an et vise à inciter le public à s'intéresser aux grands créateurs de musique contemporaine de notre pays. La SMCQ chapeaute le tout, couvrant ainsi un corps pluriel constitué d'une multitude de diffuseurs partenaires. Ainsi, au cours de la saison 2011-2012, plus de 200 événements ont été présentés autour de l'œuvre d'Ana Sokolovićr. Le bilan de l'événement est impressionnant: 2749 spectateurs ont assisté à des productions ou coproductions de la SMCQ, alors que 10048 de plus ont assisté à des événements réalisés par des organismes partenaires. En outre, la Série hommage a proposé 199 activités pour la jeunesse qui ont impliqué pas moins de 21051 élèves. Au total, plus de 53000 personnes ont participé à au moins un événement dans le cadre de cette Série hommage². À l'heure actuelle où la musique classique (pour ne pas parler de la musique contemporaine...) jouit de moins en moins de présence dans l'espace public - et notamment à la radio d'État - , ces chiffres ont de quoi surprendre.

Parmi les organismes partenaires de la SMCQ, la revue Circuit a été présente dès le début. En effet, tous les deux ans, Circuit consacre l'un de ses numéros à un portrait du créateur célébré par la Série hommage. Ainsi, le vol. 18, nº 3 (2008) a été dédié à Claude Vivier et le vol. 20, nº 3 (2010) à Gilles Tremblay. Et voilà que le numéro que vous tenez entre vos mains explore l'univers onirique d'Ana Sokolović. Nous profitons de l'occasion pour ajouter

1. Le site Internet de la SMCQ offre une liste complète des concerts présentés dans le cadre de sa Série hommage à Ana Sokolovic: $<$ www.smcq.qc.ca/smcq/fr/ hommage/2011/?page=2> (consulté le 12 novembre 2012).

2. Information fournie par l'administration de la SMCQ. 
3. Un chapitre du film Composer?!, réalisé par Anne Marie Messier et Irène Messier, DVD, SMCQ, Ès Arts et Productions Énéri, 2006. qu'en 2013-2014, ce sera au tour du compositeur Denis Gougeon de voir son travail honoré dans le cadre de la quatrième édition de la Série hommage, et, vous l'aurez deviné, Circuit dédiera le premier numéro du volume 24 à l'univers sonore de Gougeon. Avis aux auteurs intéressés!

Ce numéro n'est pourtant pas seulement un hommage à Ana Sokolović: cette compositrice à la quarantaine tout juste passée et dont l'opéra Midnight Court a été présenté au Covent Garden de Londres en 2006, a-t-elle réellement besoin d'un hommage de notre part? La réponse est non, et cela vaut autant pour Claude Vivier ou Gilles Tremblay qui n'ont pas non plus besoin de cette reconnaissance modeste que nous sommes en mesure de leur offrir. La revue Circuit est avant tout un espace où peut circuler librement un discours soutenu sur la musique qui nous émerveille, surtout celle de notre temps et de notre lieu. Telle est la vocation que se donne ce numéro dans le cas de Sokolović. Bien plus qu'un simple «hommage à... », les pages suivantes se veulent également un "portrait de...", voire une «carte blanche à...» Ana Sokolović, puisque la compositrice a été impliquée, discrètement mais de façon décisive, dans la genèse de ce numéro, notamment en choisissant les illustrations de Jaber Lutfi qui l'ornent (dont certains tableaux sont accrochés chez elle...). Mais surtout, ce numéro est une invitation à découvrir un monde sonore ludique, féerique, tout aussi plurisémantique que polyglotte, un monde que Tamara Bernstein qualifie d' «univers où les mots peuvent se détacher de leur sens pour devenir du son pur; où les voix de femmes ramènent le mot "enchantement" à ses sources anciennes ». Également dans ce numéro, vous trouverez un article d'Anne Marie Messier, qui, ayant déjà coréalisé un film documentaire sur Sokolovićs signe ici un texte sur la jeunesse en Serbie et les années de formation de la compositrice, une histoire qui se déroule tant au Canada qu'en ex-Yougoslavie. Un entretien réalisé par la compositrice Isabelle Panneton s'intéresse au rapport entre compositeur et interprète, à la question de la dramaturgie véhiculée par la forme, et aux processus de création sollicités dans quelques œuvres récentes de Sokolović. Enfin, dans le Cahier d'analyse, Brian Harman se penche sur une des premières œuvres qui a révélé Sokolović au public montréalais, Géométrie sentimentale (1997), en proposant une sorte d'herméneutique de cette œuvre aussi riche en allusions folkloriques qu'en structuration abstraite. Le dossier thématique est agrémenté d'un catalogue d'œuvres d'Ana Sokolović mis à jour après la Série hommage, qui a bien sûr donné lieu à quelques créations. 
Enfin, signalons en passant que ce numéro prolonge l'intérêt dont Circuit a fait preuve à différents moments pour la démarche de femmes créatrices, toutes tendances esthétiques et époques historiques confondues, de Michèle Reverdy à Marcelle Deschênes, de Violet Archer à Barbara Pentland, en passant par Micheline Coulombe Saint-Marcoux et Jean Coulthardt. Ici encore, comme nous l'avons fait pour le vol. 19, $\mathrm{n}^{\circ} 1$, nous sommes ravis de consacrer un numéro à la démarche d'une compositrice.

En ce qui concerne les éléments hors thème de ce numéro, vous trouverez dans la rubrique Actualités la deuxième chronique proposée par l'équipe du site Internet Cette ville étrange, dans laquelle Julien Bilodeau signe un essai sur l'œuvre du compositeur Serge Provost. La chronique «Créé dans LeVivier », préparée par Cléo Palacio-Quintin, revient ici également pour signaler les créations de la saison 2011-2012 par les organismes partenaires du Vivier. Outre quelques nouveautés en bref concoctées par Claudine Caron et Cléo Palacio-Quintin, un compte rendu de livres réalisé par cette dernière et un texte de Barah Héon-Morissette écrit à l'occasion des vingt ans de Réseaux des arts médiatiques, signalons un texte de Louise Bail qui souligne la carrière hors pair de la musicologue Maryvonne Kendergi, qui nous a quitté l'an dernier après avoir œuvré en faveur de la création musicale au Québec pendant plus d'un demi-siècle.

Bonne lecture!

Montréal, 12 novembre 2012
4. Dans Circuit, vol. 19, nº 1 (2009), "Composer au féminin". 